\title{
Stress-Diffusion Coupling and Viscoelastic Effects on Early Stage Spinodal Decomposition in Polymer Solutions
}

\author{
Mikihito TakenakA*, Nobuyuki Toyoda, Shin Saito, and Takeji Hashimoto \\ Department of Polymer Chemistry, Graduate School of Engineering, Kyoto University, Kyoto 615-8510, Japan \\ *Corresponding author: takenaka@alloy.polym.kyoto-u.ac.jp \\ Received February 28, 2006; Accepted March 27, 2006 \\ (C) 2006 The Society of Rubber Industry, Japan
}

\begin{abstract}
We investigated the viscoelastic effects on the early stage spinodal decomposition (SD) in semidilute polymer solutions where the coupling of stress and diffusion plays an important role. The so-called viscoelastic length $\xi_{\mathrm{ve}}$, within which the stress suppresses the growth of the concentration fluctuations, was quantitatively evaluated experimentally. The evaluated value was found out to become as large as $9.0 \times 10^{2} \mathrm{~nm}$. The obtained value $\xi_{\text {ve }}$ agrees well with that independently estimated from the diffusion and viscoelasticity measurements, indicating that the DoiOnuki theory can well describe the viscoelastic effects on the dynamics of the early stage SD.
\end{abstract}

Keywords Viscoelastic effects, Spinodal decomposition, Semidilute polymer solutions, Doi-Onuki theory

\section{Introduction}

Dynamics of phase separation processes have been widely investigated for various systems such as metal alloys, binary simple liquids, and polymer blends ${ }^{1-5}$. Recently, it has been found that the dynamical asymmetry between constituent components in mixtures causes the coupling between stress and diffusion due to the asymmetric stress division ${ }^{6}$. This coupling effect causes unique "viscoelastic effects" on the phase separation process $^{7-10)}$, shear-induced concentration fluctuations or phase separation in polymer solutions ${ }^{11-14)}$ and non-singleexponential decay in dynamic light scattering (DLS) for polymer solutions ${ }^{15}$. Theoretically, Doi and Onuki (DO) have taken this effect into account by formulating the Time-Dependent-Ginzburg-Landau (TDGL) type dynamical equation which incorporates the dynamical coupling between stress and diffusion ${ }^{6}$. Onuki and Taniguchi further developed the linearized dynamical equation to apply to the early stage spinodal decomposition (SD) ${ }^{16)}$.

This coupling effect is anticipated to be a key physical factor encountered universally in the phase separation processes of various kinds of complex fluids such as gels, colloidal suspensions, and etc. Moreover, the elastic effects due to the lattice misfits between each component in metal alloys ${ }^{17,18)}$ during phase separation are considered to be one of the extreme case of the viscoelastic effects. Thus, the viscoelastic effects are thought to be the universal features in dynamically asymmetric mixtures, and the DO theory is considered o pioneer a new paradigm beyond the Cahn-Hilliard theory ${ }^{19)}$. Therefore, in this paper, we aim to unveil the effect experimentally, using semidilute polymer solutions as a model system. In more detail we aim to quantitatively explore the effect on SD in semidilute polymer solutions and to analyze the experimental results in the context of the DO theory in order to clarify how the viscoelasticity affects the growth rate of the concentration fluctuations of the system composed of polymer and solvent as dynamically asymmetric elements.

In order to appraise validity of the DO theory, we focused on so called "viscoelastic length" $\xi_{\mathrm{ve}}$, which characterizes the characteristic length within which the stress built-up on temporary entangled polymer network (as a consequence of the growth of the concentration fluctuations) and resulting stress relaxation process suppresses the growth rate of the concentration fluctuation. This length brings about the $\mathrm{q}^{-2}$ dependence of Onsager coefficient at $\mathrm{q} \xi_{\mathrm{ve}}>1$. Here $\mathrm{q}$ denotes wave number for a particular Fourier mode of the concentration fluctuations which is detected at a particular magnitude of a scattering vector $\mathrm{q}[=(4 \pi / \lambda) \sin (\theta / 2)$ with $\lambda$ and $\theta$ being, respectively, the wavelength of the incident beam and the scattering angle in the solution] in the scattering experiment. $\xi_{\mathrm{ve}}$ becomes larger as the dynamical asymmetry between constituent components in a mixture 
increases and can be much larger than the radius of gyration $\mathrm{R}_{\mathrm{g}}$ of polymers in the solution. This viscoelastic length is regarded as one of characteristic lengths in the hierarchical structures of complex fluids. A uniqueness of this effect may be stressed in such a point that it depends on dynamical properties such as diffusivities and viscoelasticities, as will be detailed later. This finding may unveil new aspects to hierarchical structures of complex fluids.

In this paper, we will evaluate $\xi_{\mathrm{ve}}$ from the following two independent experiments: (i) from the time-resolved light scattering (TRLS) experiment for the early stage SD of the polymer solution and (ii) from the measurement of the diffusion and viscoelastic properties of the same solutions as that used in (i) in one phase region. Then, we will appraise the validity of the DO theory by comparing the two independent results of $\xi_{\mathrm{ve}}$.

\section{Experimental}

The polymer used here is polystyrene (PS) with the weight-averaged molecular weight $\mathrm{M}_{\mathrm{w}}$ of $5.48 \times 10^{6}$ and the polydispersity index $M_{w} / M_{n}=1.15$, where $M_{n}$ denotes the number-averaged molecular weight. Dioctyl Phthalate (DOP) was used as a solvent. The concentration of PS used was $6.0 \mathrm{wt} \%$ which corresponds to 6.7 times as much as the overlap concentration. The PS/DOP solution has an upper critical solution temperature type phase diagram with cloud point of $13.8^{\circ} \mathrm{C}$.

We quenched the solution from $20.0^{\circ} \mathrm{C}$ to $12.3^{\circ} \mathrm{C}$ and then measured the change in the scattered intensity $\mathrm{I}(\mathrm{q}, \mathrm{t})$ as a function of $\mathrm{q}$ and time $\mathrm{t}$ during the phase separation process of the solution with TRLS technique with $\mathrm{He}-\mathrm{Ne}$ laser as a light source. We measured the diffusional characteristics with DLS and viscoelasticities with the stress relaxation measurement (SRM) and steady-shear viscosity measurement (SVM), all three in the one phase region of the same solution as that used for the TRLS measurement. The instrument used for DLS was ALV$5000^{\circledR}$ with an $\mathrm{Ar}^{+}$ion laser as a light source. A rheometer (Rheometircs $\mathrm{ARES}^{\circledR}$ ) with a fluid bath temperature control was used for SRM and SVM with the cone-andplate fixture.

\section{Results and Discussion}

Figure 1(a) shows the change in $I(q, t)$ during the phase separation process after the onset of the quench. As time elapses, the scattered intensity increases with time in the observed q-range, and the scattering peak appears at
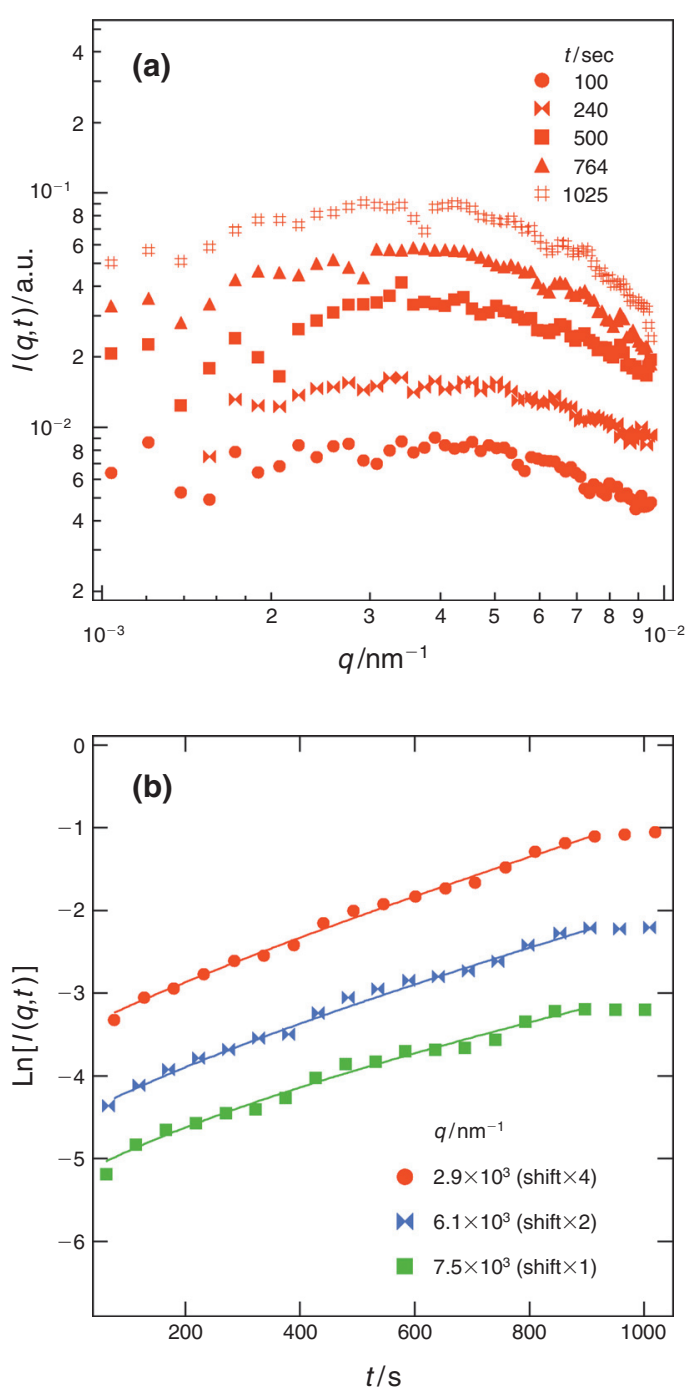

Figure 1. Changes in the light scattering profiles with time during an early stage phase-separation process after the quench from $\mathrm{T}=20.0$ to $12.3^{\circ} \mathrm{C}$.

$\mathrm{q} \cong 4.0 \times 10^{-3} \mathrm{~nm}^{-1}$. This change in $\mathrm{I}(\mathrm{q}, \mathrm{t})$ is similar to that in the early stage spinodal decomposition in polymer blends or dilute polymer solutions except for the fact that the peaks here are much broader than others. It is well confirmed $^{20-24)}$ that the dynamics of the early stage SD can be approximated by Cahn-Hilliard-Cook theory (CHC theory ${ }^{19,25,26)}$. According to the $\mathrm{CHC}$ theory, the change in the $\mathrm{q}$-Fourier mode of the scattered intensity $\mathrm{I}(\mathrm{q}, \mathrm{t})$ at time $\mathrm{t}$ can be described by the following equation:

$$
\mathrm{I}(\mathrm{q}, \mathrm{t})=\mathrm{I}(\mathrm{q}, \infty)+[\mathrm{I}(\mathrm{q}, 0)-\mathrm{I}(\mathrm{q}, \infty)] \exp [2 \mathrm{R}(\mathrm{q}) \mathrm{t}],
$$

where $\mathrm{I}(\mathrm{q}, 0), \mathrm{I}(\mathrm{q}, \infty)$, and $\mathrm{R}(\mathrm{q})$ are, respectively, $\mathrm{I}(\mathrm{q}, \mathrm{t})$ at $\mathrm{t}=0$, the virtual structure factor due to the random thermal force effects, and the growth rate. We were able to fit the 
changes in $\mathrm{I}(\mathrm{q}, \mathrm{t})$ with $\mathrm{t}$ at various fixed q's after the onset of the quench well with Eq. (1) and obtained the growth rate $\mathrm{R}(\mathrm{q})$. Figure $2(\mathrm{a})$ shows the $\mathrm{q}$-dependence of $\mathrm{R}(\mathrm{q})$. The plot has the peak at $\mathrm{q}=4.0 \times 10^{-3} \mathrm{~nm}^{-1}$ which agrees with the peak position in $\mathrm{I}(\mathrm{q}, \mathrm{t})$. The $\mathrm{CHC}$ theory gives

$$
\mathrm{R}(\mathrm{q})=\Lambda(\mathrm{q}) \mathrm{q}^{2}\left(\mathrm{r}_{0}-\mathrm{Cq}^{2}\right)
$$

Here $\Lambda(q)$ is the Onsager kinetic coefficient, $C$ is a positive constant, and $r_{0}$ is positive in unstable region and increases with the quench depth. Since the q-region covered by the light scattering experiment is much smaller than the inverse of the radius of gyration of the polymer chain $\mathrm{R}_{\mathrm{g}}{ }^{-1}$, it is expected that $\Lambda(\mathrm{q})$ is governed by the diffusion of centers of mass of polymer coils, so that $\Lambda(q)=\Lambda(0)$, a constant independent of $q$. Thus the plot of $\mathrm{R}(\mathrm{q}) / \mathrm{q}^{2}$ vs $\mathrm{q}^{2}$ should be linear. However, as shown in Figure 2(b), the plot of $\mathrm{R}(\mathrm{q}) / \mathrm{q}^{2}$ vs $\mathrm{q}^{2}$ does not show the linear relationship but rather a quite remarkable nonlinear behavior as characterized by large downward curvature, in contrast with the $\mathrm{CHC}$ prediction and the previous results $^{20-24)}$

We investigated possibility that this curvature in the plot of $\mathrm{R}(\mathrm{q}) / \mathrm{q}^{2} v s \mathrm{q}^{2}$ originates from the viscoelastic effects. Thus we analyzed the data with the DO theory. According to the DO theory, the linearized dynamical equation for the q-Fourier mode of the local concentration fluctuations of polymer $\delta \phi_{\mathrm{A}}(\mathrm{q}, \mathrm{t})$ at $\mathrm{t}$ is given by

$$
\begin{gathered}
\frac{\partial}{\partial \mathrm{t}} \delta \phi_{\mathrm{A}}(\mathrm{q}, \mathrm{t})=-\Lambda(0) \mathrm{q}^{2}\left(-\mathrm{r}_{0}+\mathrm{Cq}^{2}\right) \delta \phi_{\mathrm{A}}(\mathrm{q}, \mathrm{t}) \\
-\frac{4 \Lambda(0) \mathrm{q}^{2}}{3 \phi_{\mathrm{A}, 0}^{2}} \int_{0}^{\mathrm{t}} \mathrm{dt}^{\prime} \mathrm{G}\left(\mathrm{t}-\mathrm{t}^{\prime}\right) \frac{\partial}{\partial \mathrm{t}^{\prime}} \delta \phi_{\mathrm{A}}\left(\mathrm{q}, \mathrm{t}^{\prime}\right) \\
+\varsigma(\mathrm{q}, \mathrm{t})
\end{gathered}
$$

where $\phi_{\mathrm{A}, 0}$ and $\mathrm{G}(\mathrm{t})$ are, respectively, the space-averaged volume fraction of the polymer in the solution and the shear stress relaxation function. $\varsigma(\mathrm{q}, \mathrm{t})$ is the $\mathrm{q}$-Fourier mode of random thermal force term given by the fluctuation-dissipation relation $^{26)}$. It is well-known that $\mathrm{G}(\mathrm{t})$ is phenomelogically expressed by a sum of fundamental relaxation processes ${ }^{27)}$ :

$$
\mathrm{G}(\mathrm{t})=\sum_{\mathrm{i}=1}^{\mathrm{n}} \mathrm{G}_{\mathrm{i}} \exp \left(-\mathrm{t} / \tau_{\mathrm{i}}\right)
$$

with $\mathrm{G}_{\mathrm{i}}$ and $\tau_{\mathrm{i}}$ being strength and relaxation time of the $\mathrm{i}$-th relaxation process where $\tau_{1}>\tau_{2} \ldots>\tau_{\mathrm{n}}$. If $\mathrm{R}(\mathrm{q})^{-1}$ is larger than the longest relaxation time $\tau_{1}$ in $\mathrm{G}(\mathrm{t})$, we can set $\partial \delta \phi_{\mathrm{A}}\left(\mathrm{q}, \mathrm{t}^{\prime}\right) / \partial \mathrm{t}^{\prime}=\partial \delta \phi_{\mathrm{A}}(\mathrm{q}, \mathrm{t}) / \partial \mathrm{t}$ so that Eq. (3) reduces to
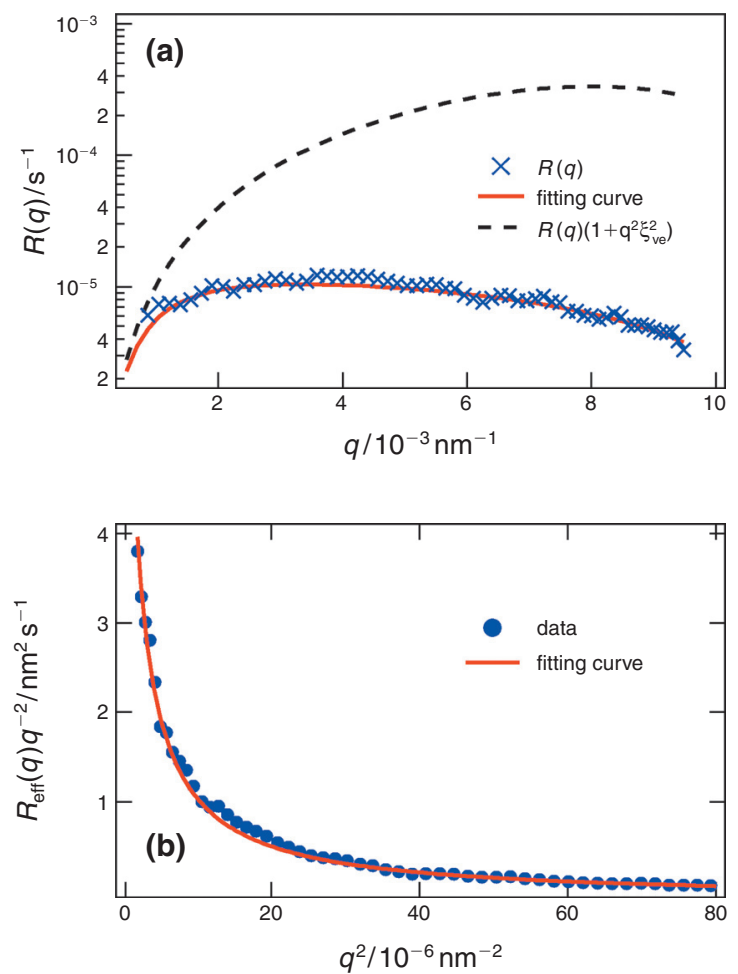

Figure 2. (a) Semi-logarithmic plots of $\mathrm{R}(\mathrm{q})$ (crosses) vs $\mathrm{q}$. The solid line indicates the R(q) calculated by using Eqs. (2) and (6) with $\xi_{\mathrm{ve}}=9.0 \times 10^{2} \mathrm{~nm}$ and the broken line the fictitious curve of $\mathrm{R}(\mathrm{q})$ with $\xi_{\mathrm{ve}}=0$. (b) $\mathrm{R}(\mathrm{q}) / \mathrm{q}^{2}$ is plotted as a function of $\mathrm{q}^{2}$. The solid line is the best fit of the results (filled circles) with Eqs. (2) and (6) with $\xi_{\mathrm{ve}}=9.0 \times 10^{2} \mathrm{~nm}$, while the broken line is the fictitious case with $\xi_{\mathrm{ve}}=0$.

$$
\begin{aligned}
& \left(1+\frac{4 \Lambda(0) \sum_{\mathrm{i}=1}^{\mathrm{n}} \mathrm{G}_{\mathrm{i}} \tau_{\mathrm{i}}}{3 \phi_{\mathrm{A}, 0}^{2}} \mathrm{q}^{2}\right) \frac{\partial}{\partial \mathrm{t}} \delta \phi_{\mathrm{A}}(\mathrm{q}, \mathrm{t}) \\
& =-\Lambda(0) \mathrm{q}^{2}\left(-\mathrm{r}_{0}+\mathrm{Cq}^{2}\right) \delta \phi_{\mathrm{A}}(\mathrm{q}, \mathrm{t})
\end{aligned}
$$

and the change in $\mathrm{I}(\mathrm{q}, \mathrm{t})\left[\sim\left\langle\left|\delta \phi_{\mathrm{A}}(\mathrm{q}, \mathrm{t})\right|^{2}\right\rangle\right.$, where \langle\rangle denotes thermal average] with time is described by the same form as Eq. (2) except for the fact that $\Lambda$ (q) in Eq. (2), which is equal to $\Lambda(0)$ in our experimental condition, should be replaced by q-dependent $\Lambda_{\text {eff }}(\mathrm{q})$ given by

$$
\Lambda_{\text {eff }}(\mathrm{q})=\frac{\Lambda(0)}{1+\xi_{\mathrm{ve}}^{2} \mathrm{q}^{2}} .
$$

The q-dependence of the Onsager coefficient originates from the viscoelastic length $\xi_{\mathrm{ve}}$ defined by 


$$
\xi_{\mathrm{ve}}=\left(\frac{4 \Lambda(0) \sum_{\mathrm{i}=1}^{\mathrm{n}} \mathrm{G}_{\mathrm{i}} \tau_{\mathrm{i}}}{3 \phi_{\mathrm{A}, 0}^{2}}\right)^{1 / 2}=\left(\frac{4 \Lambda(0) \eta_{0}}{3 \phi_{\mathrm{A}, 0}^{2}}\right)^{1 / 2},
$$

where $\eta_{0}$ is zero-shear viscosity of the solution.

In this experimental condition, the observed minimum value of $\mathrm{R}(\mathrm{q})^{-1}$ is $8.3 \times 10^{4} \mathrm{~s}$ and much longer than $\tau_{1}=270$ s so that the assumption leading to Eq. (6) is quite legitimate. We were able to fit the data of $\mathrm{R}(\mathrm{q}) / \mathrm{q}^{2} v s \mathrm{q}^{2}$ plot with Eqs. (2) and (6) having $\mathrm{r}_{0}, \mathrm{C}$, and $\xi_{\mathrm{ve}}$ as floating parameters, as shown by the solid line in Figure 2(b), and obtained $\xi_{\mathrm{ve}}=9.0 \times 10^{2} \mathrm{~nm}$. Figure 3 shows the qdependence of $\Lambda_{\text {eff }}(\mathrm{q}) . \Lambda_{\text {eff }}(\mathrm{q})$ decreases with a power law of $\mathrm{q}^{-2}$ at a high q-region, indicating that the viscoelastic effects very much suppress the growth rate of the concentration fluctuations at the high q-region and can cause the downward curvature in the plot shown in Figure 2(b). The broken line in Figures 2(a), 2(b), and 3 present the case where the viscoelastic effects on $\mathrm{R}(\mathrm{q})$ could be ignored $\left(\xi_{\mathrm{ve}}=0\right)$. In comparison between two cases, the actual growth rate $\mathrm{R}(\mathrm{q})$ is much lower than the fictitious growth rate with $\xi_{\mathrm{ve}}=0$ and the peak position of the fictitious $\mathrm{R}(\mathrm{q})$ with $\xi_{\mathrm{ve}}=0$ is $8.0 \times 10^{-3} \mathrm{~nm}^{-1}$, about twice as large as that of the actual $\mathrm{R}(\mathrm{q})$. This indicates that the viscoelastic effects affect not only the growth rate of the concentration fluctuations but also the wavelength of the initial periodic structure developed in the early stage SD.

In order to confirm validity of $\xi_{\mathrm{ve}}$ obtained above on the basis of the DO theory, we aim to check whether or not the value $\xi_{\mathrm{ve}}$ agrees with that estimated from other independent experiments. $\xi_{\mathrm{ve}}$ can be estimated from Eq. (7), provided that the values of $\eta_{0}$ and $\Lambda(0)$ can be measured. $\eta_{0}$ can be obtained straightforwardly from the viscoelastic measurement.

$\Lambda(0)$ can be estimated from the two characteristic parameters, inter-diffusion coefficient $\mathrm{D}$ and cooperative diffusion coefficient $\mathrm{D}_{\mathrm{c}}$, again in the context of the DO theory ${ }^{6}$,

$$
\Lambda(0)=\frac{3 \phi_{\mathrm{A}, 0}^{2}\left(\mathrm{D}_{\mathrm{c}}-\mathrm{D}\right)}{4 \sum_{\mathrm{i}=1}^{\mathrm{n}} \mathrm{G}_{\mathrm{i}}} .
$$

Here $\sum_{i=1}^{n} G_{i}$ corresponds to the rubbery plateau modulus of the solution, which can be estimated from the viscoelastic measurement. Now the characteristic parameters $\mathrm{D}$ and $\mathrm{D}_{\mathrm{c}}$ can be obtained as follows from the

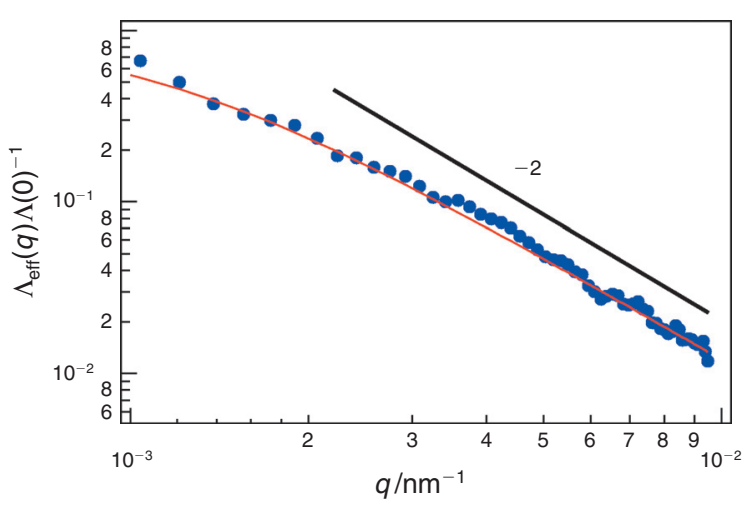

Figure 3. $\Lambda_{\text {eff }}(q) / \Lambda(0)$ is plotted as a function of $q$. The solid line indicates $1 /\left(1+\mathrm{q}^{2} \xi_{\mathrm{ve}}{ }^{2}\right)$ with $\xi_{\mathrm{ve}}=9.0 \times 10^{2} \mathrm{~nm}$, while the broken line is the fictitious case with $\xi_{\mathrm{ve}}=0$.

dynamic structure factor $\mathrm{S}(\mathrm{q}, \mathrm{t})$ with DLS in one phase region, again based on the DO theory.

Theoretically, $\mathrm{S}(\mathrm{q}, \mathrm{t})$ affected by the viscoelastic effects is expressed by

$$
\mathrm{S}(\mathrm{q}, \mathrm{t})=\sum_{\mathrm{i}=1}^{\mathrm{m}} \mathrm{S}_{\mathrm{i}} \exp \left[-\Gamma_{\mathrm{i}}(\mathrm{q}) \mathrm{t}\right],
$$

where $\Gamma_{i}$ is the relaxation rate and $S_{i}$ the relaxation intensity of the i-th relaxation process. $\Gamma_{\mathrm{i}}$ and $\mathrm{S}_{\mathrm{i}}$ have the following relationship with $\mathrm{D}$ and $\mathrm{D}_{\mathrm{c}}$ :

$$
\sum_{i=1}^{m} S_{i} \Gamma_{i}(q)=D q^{2}
$$

and

$$
\sum_{i=1}^{m} \Gamma_{i}(q)=D_{c} q^{2}+\sum_{j=1}^{n} \tau_{j}^{-1}
$$

Thus, $\mathrm{D}$ and $\mathrm{D}_{\mathrm{c}}$ can be, respectively, estimated from the slope of $\sum_{\mathrm{i}=1}^{\mathrm{m}} \mathrm{S}_{\mathrm{i}} \Gamma_{\mathrm{i}} v s \mathrm{q}^{2}$ plot, and from the slope of $\sum_{\mathrm{i}=1}^{\mathrm{m}} \Gamma_{\mathrm{i}}$ vs $\mathrm{q}^{2}$ plot $^{28)}$. Moreover $\sum_{\mathrm{i}=1}^{\mathrm{m}} \mathrm{S}_{\mathrm{i}} \Gamma_{\mathrm{i}}$ can be estimated from the first cumulant $\mathrm{K}_{1}(\mathrm{q})=-[\partial \ln \mathrm{S}(\mathrm{q}, \mathrm{t}) / \partial \mathrm{t}]_{\mathrm{t} \rightarrow 0}$ of $\mathrm{S}(\mathrm{q}, \mathrm{t})$, since $\sum_{\mathrm{i}=1}^{\mathrm{m}} \mathrm{S}_{\mathrm{i}} \Gamma_{\mathrm{i}}=\mathrm{K}_{1}$. As for the estimation of $\mathrm{D}_{\mathrm{c}}$, we first analyzed the measured $\mathrm{S}(\mathrm{q}, \mathrm{t})$ with $\mathrm{CONTIN}^{29)}$ to obtain the distribution of $\Gamma_{\mathrm{i}}$ and then the value $\sum_{\mathrm{i}=1}^{\mathrm{m}} \Gamma_{\mathrm{i}}$. Since we can not directly measure $\mathrm{D}_{\mathrm{c}}, \mathrm{D}, \eta_{0}$, and $\sum_{\mathrm{i}=1}^{\mathrm{n}} \mathrm{G}_{\mathrm{i}, 0}$ at $12.3^{\circ} \mathrm{C}$ which corresponds to the temperature of our the TRLS measurement in the two phase region, we extrapolated the 
corresponding values of $D_{c}, D, \eta_{0}$, and $\sum_{i=1}^{n} G_{i, 0}$ measured at various temperatures in one phase region.

The temperature dependence of $\mathrm{D}_{\mathrm{c}}-\mathrm{D}$ thus estimated had the following Arrenius type equation:

$$
\mathrm{D}_{\mathrm{c}}-\mathrm{D}=3.00 \times 10^{2} \exp \left(-8.24 \times 10^{3} / \mathrm{T}\right)
$$

and the extrapolated value of $\mathrm{D}_{\mathrm{c}}-\mathrm{D}$ at $12.3^{\circ} \mathrm{C}\left(\mathrm{T}^{-1}=\right.$ $3.50 \times 10^{-3} \mathrm{~K}^{-1}$ ) is $8.87 \times 10^{-11} \mathrm{~cm}^{2} / \mathrm{s} . \quad \eta_{0}$ was estimated from the SVM for the same solution as a function of T, the result of which was expressed by

$$
\eta_{0}=8.51 \times 10^{-8} \exp \left[\left(6.91 \times 10^{3}\right) / \mathrm{T}\right],
$$

and $\eta_{0}=2.73 \times 10^{3} \mathrm{~Pa} \cdot \mathrm{s}$ at $12.3^{\circ} \mathrm{C}$. We also estimated $\sum_{i=1}^{n} G_{i, 0}=26.8 P a$ from the SRM of the same solution.

$\xi_{\mathrm{ve}}$ calculated from the values $\mathrm{D}_{\mathrm{c}}-\mathrm{D}, \eta_{0}$, and $\sum_{i=1}^{n} G_{i, 0}$ thus obtained is $9.6 \times 10^{2} n m$ and well in agreement with that obtained from the dynamics of early stage SD. This agreement is the first clear-cut confirmation of the fact that the DO theory can well describe quantitatively the dynamics of the phase separation process in dynamically asymmetric binary systems. The temperature dependence of $\xi_{\mathrm{ve}}$ is described elsewhere ${ }^{30)}$.

In summary, we investigated the viscoelastic effects on the early stage SD in the PS/DOP solution. We found that the effects strongly suppress the growth rate of the concentration fluctuations and that the viscoelastic length can be very large, $\xi_{\mathrm{ve}}=9.0 \times 10^{2} \mathrm{~nm} \cong 13 \mathrm{R}_{\mathrm{g}}$. The value $\xi_{\mathrm{ve}}=9.0 \times 10^{2} \mathrm{~nm}$ obtained in the early stage SD well agrees with $\xi_{\mathrm{ve}}=9.5 \times 10^{2} \mathrm{~nm}$ estimated from the diffusion and viscoelasticity measurements. This agreement confirms validity of the DO theory.

Acknowledgment This work was financially supported in part by a Grant-in-Aid for Scientific Research (under Grant No. 12640392, 13031053 and 14045245) from the
Ministry of Education, Science, and Culture, Japan.

\section{References}

1) Gunton J. D., Miguel M. S., Sahni P. S.: Phase Transitions, 8 , 269 (1983).

2) Hashimoto T.: Phase Transitions, 12, 47 (1988).

3) Hashimoto T.: "Dynamics of Ordering Processes in Condensed Matter", Ed. Komura S., Furukawa H., Plenum Publishing, New York, (1988), p. 421.

4) Binder K.: "Phase Transformations in Materials", Ed. Haasen P., VCH, Weinheim, (1990), Vol. 5, p. 405.

5) Hashimoto T.: "Structure and Properties of Polymers", Ed. Thomas E. L., VCH, Weinheim, (1993), p. 252.

6) Doi M. and Onuki A.: J. Phys. II (France), 2, 1631 (1992).

7) Tanaka H.: Phys. Rev. Lett., 71, 3158 (1993).

8) Tanaka H.: J. Chem. Phys., 100, 5323 (1994).

9) Tanaka H.: Phys. Rev. Lett., 76, 787 (1996).

10) Tanaka H.: Phys. Rev. E, 56, 4451 (1997).

11) Hashimoto T., Fujioka K.: J. Phys. Soc. Jpn., 60, 356 (1991).

12) Dixon P. K., Pine D. J., Wu X.-L.: Phys. Rev. Lett., 68, 2239 (1992).

13) Kume T., Hattori T., Hashimoto T.: Macromolecules, 30, 427 (1997).

14) Saito S., Matsuzaka K., Hashimoto T.: Macromolecules, 32, 4879 (1999).

15) Wu X.-L., Pine D. J., Dixon P. K.: Phys. Rev. Lett., 66, 2408 (1991).

16) Onuki A., Taniguchi T.: J. Chem. Phys., 106, 5761 (1997).

17) Cahn J. W.: Acta Metall., 32, 1925 (1964).

18) Miyazaki T., Doi M.: Mater. Sci. Eng., A10 (1989).

19) Cahn J. W.: J. Chem. Phys., 42, 93 (1965).

20) Hashimoto T., Kumaki J., Kawai H.: Macromolecules, 16, 641 (1983).

21) Hashimoto T., Itakura M., Hasegawa H.: J. Chem. Phys., 85, 6118 (1986).

22) Bates F. S., Wiltzius P.: J. Chem. Phys., 91, 3258 (1989).

23) Higgins J. S., Fruitwala H. A., Tomlins P. E.: Br. Polym. J., 21, 247 (1989).

24) Takenaka M., Hashimoto T.: J. Chem. Phys., 96, 6177 (1992).

25) Cook H. E.: Acta Met., 18, 297 (1970).

26) Binder K.: J. Chem. Phys., 79, 6387 (1983).

27) Ferry J. D.: "Viscoelastic Properties of Polymers". Willey, New York, (1980).

28) Einaga Y. and Karube D.: Polymer, 40, 157 (1998).

29) Provencher S. W.: Macromol. Chem., 180, 201 (1979).

30) Toyoda N., Takenaka M., Saito S., Hashimoto T.: Polymer, $\mathbf{4 2}$, 9193 (2001). 